\title{
NGC 2146's starburst region and extended structure
}

\author{
A. Greve ${ }^{1}$, N. Neininger ${ }^{2}$, A. Sievers ${ }^{3}$, and A. Tarchi ${ }^{4,5}$ \\ 1 IRAM, 300 rue de la Piscine, 38406 St.-Martin-d'Hères, France \\ e-mail: greve@iram.fr \\ 2 Astronomisches Institut der Universität Bonn, 71 Auf dem Hügel, 53121 Bonn, Germany \\ 3 IRAM, Nucleo Central, 7 Avenida Divina Pastora, 18012 Granada, Spain \\ 4 INAF - Instituto di Radioastronomia, via Gobetti 101, 40129 Bologna, Italy \\ 5 INAF - Osservatorio Astronomico di Cagliari, Loc. Poggio dei Pini, Strada 54, 09012 Capoterra (CA), Italy
}

Received 24 June 2005 / Accepted 26 July 2006

\section{ABSTRACT}

\begin{abstract}
We present mm-wavelength and optical observations of the starburst region in NGC 2146. This region of $\sim 4$ kpc diameter contains a well-ordered distribution of stars, gas, and dust, and a well-ordered rotation. The possible anomalies found in our observation are a warped CO distribution and an increase in the CO line width at the NW edge of the starburst region. The investigation of a possible encounter or merger origin of the starburst may therefore have to concentrate on the distorted outer structure of the galaxy.

There are three large-scale features of $\sim 20 \mathrm{kpc}$ extent, two of which give the impression of being stellar sub-systems. The third feature is an incomplete and expanding ring of $\mathrm{H}$ II regions and stars, apparently indicating an event that occurred some $300 \mathrm{Myr}$ ago.
\end{abstract}

Key words. galaxies: general - galaxies: starburst

\section{Introduction}

The starburst galaxy NGC 2146 of type Sb II pec (Sandage \& Tammann 1987), at a distance of $14.5 \mathrm{Mpc}$ (with $1^{\prime \prime} \approx 70 \mathrm{pc}$ ), is large and massive and has some outstanding features: a distorted outer shape, although three spiral arms seem to be distinguishable (de Vaucouleurs 1950; Burbidge et al. 1959; Benvenuti et al. 1975); H I tails, at least at the southern side of an extension up to $150 \mathrm{kpc}$ (Caspers \& Shane 1986; Taramopoulos et al. 2001); a large amount of dust concentrated near the center, with part of it visible as a prominent dust lane and dust "fingers" and polarisation of the visible light (Benvenuti et al. 1975; Sandage \& Bedke 1994; Draper et al. 1995); it has relatively high infrared luminosity $L_{\mathrm{IR}}=6 \times 10^{10} L_{\odot}$ (Sanders et al. 1986); a central condensation, a weak ring, and extended and warped emission of molecular gas (CO) inside which the starburst occurs (Jackson \& Ho 1988; Young et al. 1988); two $\mathrm{H}_{2} \mathrm{O}$ kilo-masers in the starburst region (Tarchi et al. 2002), along with radio supernovae, supernova remnants, and ultra-dense H II regions (Kronberg \& Biermann 1981; Tarchi et al. 2000); strong nonthermal continuum radiation produced to a large extent by supernova explosions (Lisenfeld et al. 1996); X-ray point sources and diffuse X-ray emission (Armus et al. 1995; Della Ceca et al. 1999; Inui et al. 2005); a cone-like outflow along the minor axis (Armus et al. 1995; Greve et al. 2000); and maybe a stellar bar (Cabrera-Lavers \& Garzon 2004). In short, NGC 2146 is a distorted, dusty, and molecule-rich galaxy with a strong central starburst and a superwind driven out along the minor axis.

However, NGC 2146 does not have an evident companion galaxy that may have induced the starburst (Fisher \& Tully 1976; for a contrary opinion see Schmitt 2001). It has therefore been proposed that NGC 2146 is a far-evolved merger, and several observations seem to substantiate this speculation; viz. the asymmetry of "spiral arm III" (de Vaucouleurs 1950; Benvenuti et al. 1975 [BCD-75]); the asymmetric, integrated H I velocity profile
(Roberts 1978); distortions of H I gas at kpc-scales (Condon et al. 1982); distortions of the $\mathrm{H} \alpha$ velocity field along the dust lane and in the outer regions of the disk; an apparently warped optical disk; a "dent" in the SE part of the rotation curve; and a $[\mathrm{NII}] / \mathrm{H} \alpha$ ratio indicating shock excitation in the NE part of the galaxy (Hutchings et al. 1990, [HEA-90]). Since a clear material and kinematic signature of such a merger is nevertheless barely visible, we may assume that the merger occurred long ago so that it is difficult to understand how it affects the ongoing starburst today. However, $N$-body gasdynamic studies by Mihos \& Hernquist (1996) and Barnes \& Hernquist (1996) have shown that a starburst can occur during the final stage of a merger when most of the merger signature has disappeared. This happens, in particular, when the host galaxy has a prominent bulge, as is perhaps the case in NGC 2146 as indicated in optical images. If we follow the numerical simulations by Mihos \& Hernquist, a comparable situation could be the starburst predicted to appear 100 Myr after a merger has occurred, and where the distribution of gas and young stars somewhat resembles that of NGC 2146 (their Fig. 4, $t \approx 80$ ). In their prediction, a few $10^{9} M_{\odot}$ of cooler gas is present at that time as a warped disk at the center of the galaxy, able to start and fuel a starburst.

Contrary to the far-evolved merger hypothesis, Taramopoulos et al. (2001) propose from their observation of a Northern and Southern H I tail $(\sim 150 \mathrm{kpc}$ extension to the South) that the most active part of an encounter has occurred $\sim 1000$ Myr ago between NGC 2146 and a gas-rich, slow rotating, low-surface-brightness (LSB) galaxy. The remaining part of this galaxy is perhaps found $\sim 70 \mathrm{kpc}$ south of NGC 2146 as an H I concentration of $\sim 1.5 \times 10^{8} M_{\odot}$. The galaxy that encountered NGC 2146 has lost a large fraction $(\sim 50 \%)$ of its gas (and stars) because of a favorable orientation of the orbital momentum and the rotation momentum of the galaxies. A large mass transfer in such an encounter with favorably oriented 
Table 1. List of radio observations.

\begin{tabular}{|c|c|c|c|c|c|c|c|}
\hline Line/Contin. & $\begin{array}{l}\text { Wavel. } \\
(\mathrm{mm})\end{array}$ & $\begin{array}{c}\text { Beam width } \\
\left({ }^{\prime \prime}\right)\end{array}$ & $\begin{array}{l}\text { PA } \\
\left({ }^{\circ}\right)\end{array}$ & $\begin{array}{c}\text { Resol. } \\
\left(\mathrm{km} \mathrm{s}^{-1}\right)\end{array}$ & $\begin{array}{l}T_{\text {sys }} \\
(\mathrm{K})\end{array}$ & $\begin{array}{c}\text { Calibration } \\
\text { sources }\end{array}$ & $\begin{array}{l}\text { Date/Quality } \\
\text { of observation }\end{array}$ \\
\hline \multicolumn{8}{|l|}{ PV } \\
\hline Continuum & 1.3 & 11 & - & - & 250 & planets $^{a}$ & 1994, good (+ repeats) \\
\hline${ }^{12} \mathrm{CO}(1-0)$ & 2.6 & 22 & - & 1.5 & $150^{b}$ & $\mathrm{HC}, \mathrm{LC}^{d}$ & 1993,1995; good \\
\hline${ }^{12} \mathrm{CO}(2-1)$ & 1.3 & 11 & - & 2.4 & $250-350^{b}$ & $\mathrm{HC}, \mathrm{LC}^{d}$ & 1993,1995; good \\
\hline \multicolumn{8}{|l|}{ PdB } \\
\hline${ }^{12} \mathrm{CO}(1-0)$ & 2.6 & $4.1 \times 3.6$ & -36 & 6.5 & $250-350^{b}$ & $\mathrm{QSO}( \pm 5 \%)^{e}$ & Nov.-96, moderate \\
\hline${ }^{12} \mathrm{CO}(2-1)$ & 1.3 & $2.8 \times 2.2$ & -77 & 3.3 & $250-350^{b}$ & $\mathrm{QSO}( \pm 10 \%)^{e}$ & and Apr.-97, good \\
\hline${ }^{13} \mathrm{CO}(1-0)$ & 2.6 & $5.2 \times 3.6$ & 88 & 6.5 & $250-350^{c}$ & $\mathrm{QSO}( \pm 10 \%)^{e}$ & Nov.-96, Apr.-97 \\
\hline
\end{tabular}

${ }^{a}$ Especially Uranus and Neptun, temperatures from Griffin \& Orton $(1993) ;{ }^{b} \mathrm{SSB}$ receiver; ${ }^{c} \mathrm{DSB}$ receiver; ${ }^{d} \mathrm{HC}=$ hot-cold-sky method and LC $=$ line calibrators (Mauersberger et al. 1989); ${ }^{e}$ QSO for amplitude and phase calibration (in brackets: accuracy of QSO flux density) 0836+710: $S(115 \mathrm{GHz})=1.15 \mathrm{Jy}, S(230 \mathrm{GHz})=0.66 \mathrm{Jy} ; 0716+714: S(115 \mathrm{GHz})=0.9 \mathrm{Jy}, S(230 \mathrm{GHz})=1.4 \mathrm{Jy}$; at the time of observation.

momenta is predicted in numerical simulations by Wallin \& Stuart (1992). Taramopoulos et al. explain the fueling of the starburst by H I clouds falling from the tail back into NGC 2146. However, a discrepancy may exist between the encounter time $\sim 1000$ Myr ago and the present starburst, unless the fallback of H I clouds has occurred relatively recently and still continues to occur.

Finally, Young et al. (1988, [YEA-88]) propose a collision of NGC 2146 with another galaxy and draw attention to a ring of $\mathrm{H}$ II regions and stars of $\sim 20 \mathrm{kpc}$ diameter that is incomplete and apparently not co-planar with the plane of the galaxy. This prominent morphologic and kinematic feature was not discussed later on, although it may reveal a significant aspect of the history of NGC 2146.

We present a study of NGC 2146 at radio and optical wavelengths in order to obtain more information on the starburst and, perhaps, also additional evidence of the evolved merger or encounter hypothesis. We made bolometer continuum observations at $1.3 \mathrm{~mm}$ wavelength $(230 \mathrm{GHz})$ and ${ }^{12} \mathrm{CO}(1-0),{ }^{12} \mathrm{CO}(2-1)$ and ${ }^{13} \mathrm{CO}(1-0)$ line observations with the Pico Veleta $30-\mathrm{m}$ telescope (PV) and the Plateau de Bure interferometer (PdB). These observations are supplemented by spectroscopic measurements at optical and near-IR wavelengths along the major axis of NGC 2146, made in service time with the $4.2 \mathrm{~m}$ William Herschel Telescope on La Palma (RGO, Spain). The observations, the calibrations, and the quality of the radio data are listed in Table 1.

We adopt 14.5 Mpc for the distance of NGC 2146 (BCD-75; see also Kobulnicky \& Gebhardt 2000). The galaxy is seen from above and close to edge-on (inclination $i \approx 60-65^{\circ}$ ). The major axis of the galaxy is oriented in the direction SE-NW $\left(\mathrm{PA} \approx 143^{\circ}\right)$.

\section{Radio observations}

The new radio data, shown in this section, consist of radio continuum and CO line observations. From these data, we derive their spatial distribution, rotation, and mass.

\section{1. $1.3 \mathrm{~mm}(230 \mathrm{GHz})$ dust continuum emission}

Continuum observations at $1.3 \mathrm{~mm}(230 \mathrm{GHz})$ and $\sim 50 \mathrm{GHz}$ bandwidth were made with the PV 30-m telescope and the MPIfR 7-channel bolometer, at a beam width of $11^{\prime \prime}$ (FWHP). The fully sampled map was made in On-Off raster mode and at $1-2$ " pointing accuracy (rms). The noise level in the final map is $4 \mathrm{mJy} /$ beam (rms).
Figure 1 shows the measured dust emission overlaid on an RGO-archive $I$-band image, selected to show that the near-IR emission is offset from the dust emission. This is interpreted as an effect of obscuration in the central region and the dust lanes. The dust emission coincides with the starburst region and with the known peak of the $\mathrm{CO}$ emission (YEA-88). The optical dust lanes do not appear in the continuum map; they are probably relatively low-mass foreground features. The distribution of the dust emission along the major axis is shown in Fig. 2 (for a similar figure see Smith et al. 1995), together with the emission from other components. The extent of the dust emission along the major axis is $\Omega_{\mathrm{S}}(1) \approx 1200 \mathrm{pc}(\mathrm{FWHP})$, for $1^{\prime \prime}$ being $\sim 70 \mathrm{pc}$.

The dust emission stronger than $10 \mathrm{mJy} /$ beam (Fig. 1) covers the area of $\sim 60^{\prime \prime} \times 30^{\prime \prime}$, or $4 \mathrm{kpc} \times 2 \mathrm{kpc}$, and has a peak flux density of $87 \mathrm{mJy} / \mathrm{beam}$ and integrated flux density of $S^{\prime}{ }_{1.3}=$ $0.46 \pm 0.025 \mathrm{Jy}$. This flux density contains a contribution from synchrotron radiation, ${ }^{12} \mathrm{CO}(2-1)$ emission, and free-free emission, the amount of which is estimated in the Appendix. The correction may be as large as $0.1 \mathrm{Jy}$ so that the corrected integrated flux density of the dust emission may be as low as $S_{1.3}=0.36 \pm 0.025 \mathrm{Jy}$.

The continuum spectrum of NGC 2146, shown in Fig. 3, contains the updated IRAS flux densities (Sanders et al. 2003; Surace et al. 2004), the $1.3 \mathrm{~mm}$ measurement, and radio data between $2.8 \mathrm{~cm}$ and $3.6 \mathrm{~m}$ wavelength (from BCD-75; and McCutcheon 1973). The flux density at $160 \mu \mathrm{m}$ of 117.8 (9.4) Jy measured by Rickard \& Harvey (1984) with KAO and a beam of 40 to $50^{\prime \prime}$ is also inserted. Unfortunately, there are no integrated flux densities of the dust emission at, for instance, $3 \mathrm{~mm}$ and $0.85 \mathrm{~mm}$ to better constrain the dust spectrum at $\mathrm{mm}-$ wavelengths. The emission at long wavelengths is synchrotron radiation, while at short wavelengths it is dust emission. The dust temperature(s) $T_{\mathrm{d}}$ that fits the observation has been estimated by trial and error by applying the dust emission relations published by Mezger et al. (1990) and a modified Planck function (with $m$ a function of the wavelength as proposed, $Z / Z_{\odot} \approx 2$ ), and by adopting $\Omega_{\mathrm{S}}(1)=1200 \mathrm{pc}$ for the extent of the dust component (Fig. 2). A satisfactory fit of the observation apparently requires two additional smaller components, of which the widths $\Omega_{\mathrm{S}}$ and temperatures $T_{\mathrm{d}}$ were again found by trial and error. The components and their gas masses obtained in this way are given in Table 2, and their spectral distribution is shown in Fig. 3. While three components are apparently required, the dominant gas mass $M_{\mathrm{g}} \approx 6 \times 10^{9} M_{\odot}$ is contained in the cool component (1) with $T_{\mathrm{d}}=21 \mathrm{~K}$. This component extends throughout the entire volume of the starburst region. 


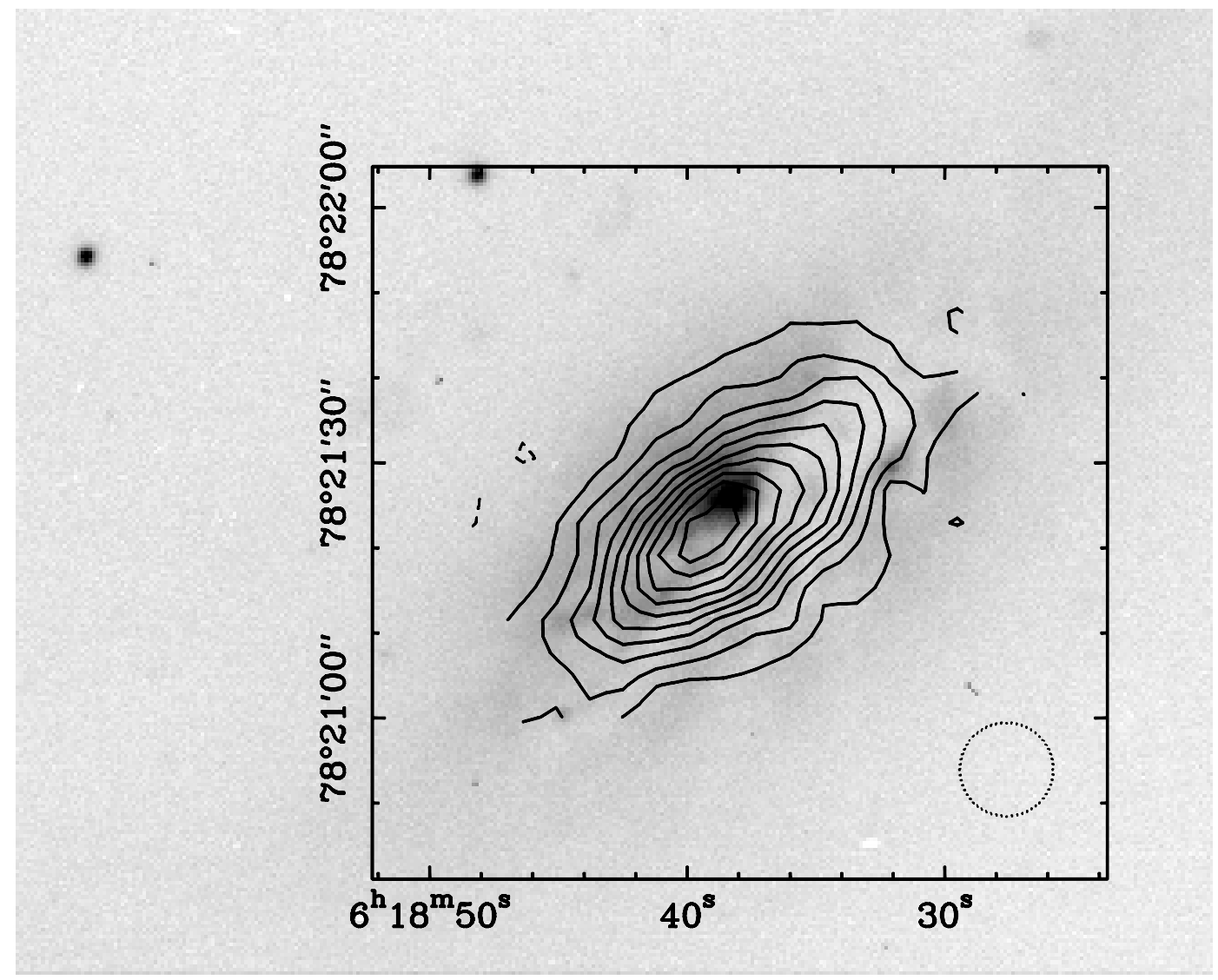

Fig. 1. $1.3 \mathrm{~mm}(230 \mathrm{GHz})$ dust emission (overlaid on an I-band image) mapped with the 7-channel MPIfR bolometer on the PV 30-m telescope of $11^{\prime \prime}$ beam width (FWHP; lower corner). Contour levels are at $-8,8,16, \ldots 80 \mathrm{mJy} / \mathrm{beam}$; the peak is at $87 \mathrm{mJy} / \mathrm{beam}$. Coordinates for J2000. The optical center appears displaced from the center of the dust emission because of extinction in the dust lanes.

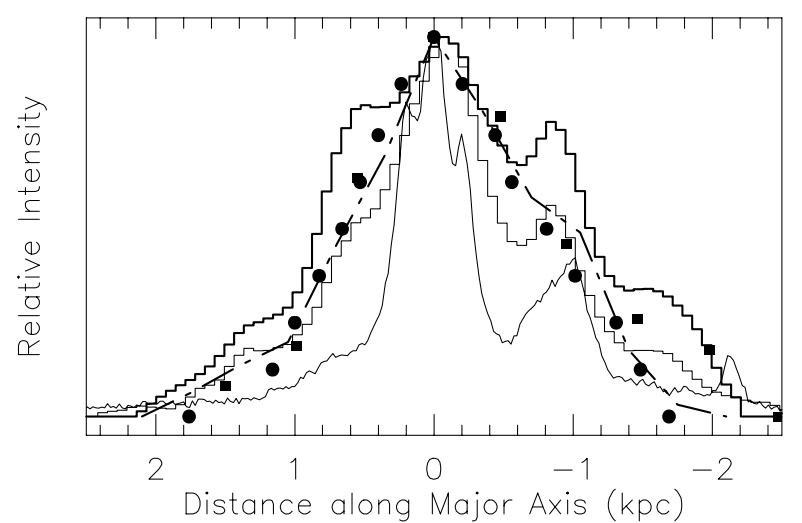

Fig. 2. Emission measured along the major axis. $1.3 \mathrm{~mm}$ dust emission: dots; $50 \mu \mathrm{m}$ dust emission (Smith et al. 1995): squares; ${ }^{12} \mathrm{CO}(1-0)$ : heavy histogram line; ${ }^{13} \mathrm{CO}(1-0)$ : thin histogram line; $\mathrm{H}_{\alpha}$ : thin line; $5 \mathrm{GHz}$ synchrotron emission (Lisenfeld et al. 1996): dashed-dotted line. $\mathrm{SE}$ is to the left, NW to the right. The intensities are normalized to the center position.

\subsection{CO observations with the PV 30-m telescope}

With the PV 30-m telescope we observed the central part of NGC 2146 in the ${ }^{12} \mathrm{CO}(1-0)$ line at $22^{\prime \prime}(115 \mathrm{GHz})$ beam width and $500 \mathrm{MHz}$ band width. Other details of the observation are listed in Table 1 . The (1-0) map is fully sampled. In the observation, the wobbler was used at $120^{\prime \prime}$ throw and $0.25 \mathrm{~Hz}$ speed, and the pointing accuracy was $1-2^{\prime \prime}(\mathrm{rms})$. At each position of the map the integration was terminated when a noise level of at least $0.1 \mathrm{~K}$ (rms) was reached at $0.6 \mathrm{MHz}$ resolution. The lowest level measured is $\sim 5 \mathrm{~K} \mathrm{~km} \mathrm{~s}^{-1}$ /beam, with the integration in velocity extending over a line width of $\sim 150$ to $200 \mathrm{~km} \mathrm{~s}^{-1}$. These values define the uncertainties quoted in Table 3.

The ${ }^{12} \mathrm{CO}(1-0)$ map measured with a beam of 22 " (FWHP) is shown in Fig. 4; a ${ }^{12} \mathrm{CO}(3-2)$ map measured with a comparable beam of 24" (FWHP) is published by Dumke et al. (2001). While the ${ }^{12} \mathrm{CO}(1-0)$ map extends over $\sim 60^{\prime \prime} \times 30^{\prime \prime}$ (at the level of $40 \mathrm{~K} \mathrm{~km} \mathrm{~s}^{-1}$ ), the ${ }^{12} \mathrm{CO}(3-2)$ distibution extends over $\sim 50^{\prime \prime} \times$ $50^{\prime \prime}$ at the same intensity level and thus seems to be more compact. From the (1-0) map of Fig. 4, we derived the ${ }^{12} \mathrm{CO}$ luminosity $L_{\mathrm{CO}}\left[\mathrm{K} \mathrm{km} \mathrm{s}^{-1} \mathrm{pc}^{2}\right]$ of the starburst region of $\sim 4 \times$ $2 \mathrm{kpc}^{2}\left(60^{\prime \prime} \times 30^{\prime \prime}\right)$ extent, listed in Table 3. The ${ }^{12} \mathrm{CO}(1-0)$ luminosity $L_{\mathrm{CO}(1-0)}$ is converted into the gas mass $M\left(\mathrm{H}_{2}\right)$ by using the relation (Sanders et al. 1991) $M\left(\mathrm{H}_{2}\right)=4.8 L_{\mathrm{CO}(1-0)}$ $\left[M_{\odot}\right]=4.6 \times 10^{9} M_{\odot}$. The gas mass derived from the dust continuum emission (Table 2) is similar to the gas mass derived from the CO line. In the following we use the $M\left(\mathrm{H}_{2}\right)$ mass.

Data of the ${ }^{12} \mathrm{CO}(2-1)$ line were taken simultaneously with the ${ }^{12} \mathrm{CO}(1-0)$ measurements, at the same spatial sampling (Fig. 4). The (2-1) map is not fully sampled. Nevertheless, the line ratio of the values of Table 3 is $R_{2,1}=(10.5 \pm 1.0) /(9.5 \pm$ $0.5)=1.10_{-0.15}^{+0.17}$ for the starburst region of $\sim 60^{\prime \prime} \times 30^{\prime \prime}$ extent. This value agrees with the value of $R_{2,1}=1.06 \pm 0.2$ measured by Aalto et al. (1995) for a central region of 28-33", and the value $R_{2,1}=0.95 \pm 0.25$ measured by Xie et al. (1994) for a central region of $45^{\prime \prime}$. 


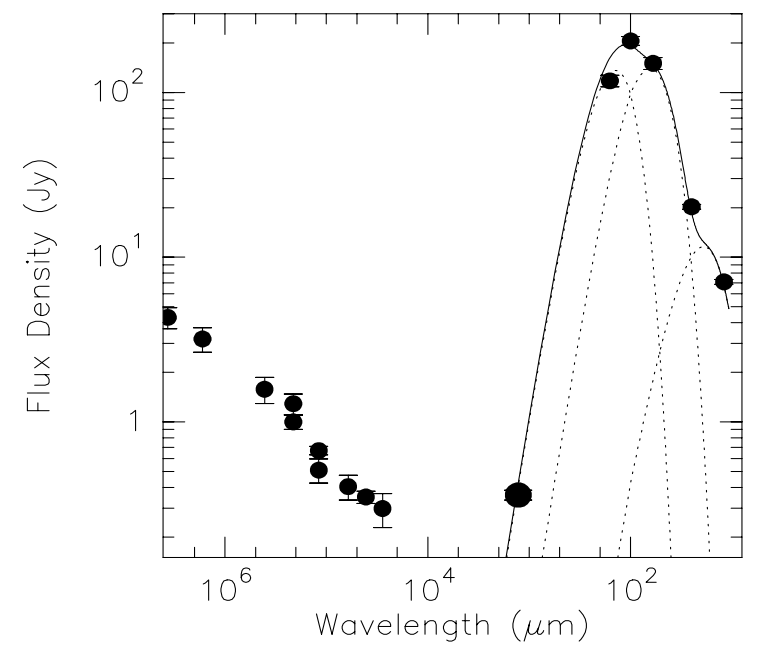

Fig. 3. Continuum spectrum from $3.6 \mathrm{~m}$ to $12 \mu \mathrm{m}$ wavelength. The data are from BCD-75, McCutcheon (1973), Rickard \& Harvey (1984), IRAS, and this paper. The emission at long wavelengths is synchrotron radiation and free-free emisssion; the emission at short wavelengths is radiation from dust. For the parameters of the dust emission components (dashed lines) see Table 2. The solid line is the sum of the components.

Table 2. Parameters of a 3-component model fit (see Fig. 3) of NGC 2146's dust emission. $\Omega_{\mathrm{s}}, T_{\mathrm{d}}$ and $M_{\mathrm{g}}$ are the source width (HPW), the dust temperature, and gas mass for $d=14.5 \mathrm{kpc}$. (The dust mass is $M_{\mathrm{d}} \approx M_{\mathrm{g}} / 100$.)

\begin{tabular}{cccc}
\hline \hline Component & $\Omega_{\mathrm{s}}(\mathrm{HPW})$ & $\begin{array}{c}T_{\mathrm{d}} \\
{[\mathrm{K}]}\end{array}$ & $\begin{array}{c}M_{\mathrm{g}} \\
{\left[M_{\odot}\right]}\end{array}$ \\
\hline 1 & $17^{\prime \prime}-1200 \mathrm{pc}$ & 21 & $(5.8 \pm 0.5) \times 10^{9}$ \\
2 & $4^{\prime \prime}-280 \mathrm{pc}$ & 52 & $\sim 7.7 \times 10^{7}$ \\
3 & $2^{\prime \prime}-140 \mathrm{pc}$ & 170 & $\sim 2.6 \times 10^{4}$ \\
\hline
\end{tabular}

\section{3. $C O$ observations with the $P d B$ interferometer}

We used 5 antennas of the PdB interferometer in a 7-mosaic observation ${ }^{1}$ of the ${ }^{12} \mathrm{CO}(1-0),{ }^{12} \mathrm{CO}(2-1)$, and ${ }^{13} \mathrm{CO}(1-0)$ line in configuration C2-D1 with baselines between $25 \mathrm{~m}$ and $180 \mathrm{~m}$. The linear dimension of the synthesized beam at the distance of NGC 2146 is $\sim 300 \mathrm{pc} \times 250 \mathrm{pc}$ for the $(1-0)$ lines and $\sim 200 \mathrm{pc} \times$ $150 \mathrm{pc}$ for the (2-1) line. For more details see Table 1.

Interferometer velocity channel maps of the ${ }^{12} \mathrm{CO}(1-0)$ line have been published before by YEA-88 (resolution $500 \mathrm{pc} \times$ $460 \mathrm{pc})$ and Jackson \& Ho (1988; resolution $450 \mathrm{pc} \times 350 \mathrm{pc})$. Our ${ }^{12} \mathrm{CO}(1-0)$ observation of higher sensitivity and higher angular resolution is very similar to these earlier observations so were not reproduced here. Some channel maps of the ${ }^{12} \mathrm{CO}(1-0)$ line are shown elsewhere (Greve et al. 2000). Figure 5 is the velocity channel map of the optically-thin ${ }^{13} \mathrm{CO}(1-0)$ emission with a noise level of $\sim 0.003 \mathrm{Jy} / \mathrm{beam}$. This map, as also for other molecular line observations, reveals the features that were already interpreted earlier as a (weak) molecular ring.

\section{The spatial distribution of $\mathrm{CO}$}

The velocity-integrated emission of the ${ }^{12} \mathrm{CO}(1-0)$ line with superimposed radio supernovae, supernova remnants, and

${ }^{1}$ The center position of the field is at RA: $6^{\mathrm{h}} 18^{\mathrm{m}} 38.6^{\mathrm{s}}$, Dec: $78^{\circ} 21^{\prime} 24.0^{\prime \prime}$ [2000]; the mosaic fields are at $(\Delta \alpha, \Delta \delta)=(25.5,-19.5)$, $(17,-13),(8.5,-6.5),(0,0),(-8.5,6.5),(-17,13),(-25.5,19.5)\left[{ }^{\prime \prime},{ }^{\prime \prime}\right]$.
Table 3. CO luminosities $L_{\mathrm{CO}}\left[\mathrm{K} \mathrm{km} \mathrm{s}^{-1} \mathrm{pc}^{2}\right]$ of the starburst region of $\sim 8 \times 10^{6} \mathrm{pc}^{2}$ area, measured with the PV 30-m telescope, and of the central region of $\sim 6 \times 10^{6} \mathrm{pc}^{2}$ area, measured with the PdB interferometer. For $d=14.5 \mathrm{Mpc}$.

\begin{tabular}{ccc}
\hline \hline Parameter & $\mathrm{PV}^{a}$ & $\mathrm{PdB}^{b}$ \\
\hline Luminosity & & \\
${ }^{12} \mathrm{CO}(1-0)$ & $(9.5 \pm 0.5) \times 10^{8}$ & $\sim 0.6 \times 10^{9}$ \\
${ }^{12} \mathrm{CO}(2-1)^{c}$ & $(10.5 \pm 1.0) \times 10^{8}$ & \\
Mass $M\left(\mathrm{H}_{2}\right)\left[M_{\odot}\right]$ & $(4.6 \pm 0.5) \times 10^{9}$ & \\
\hline
\end{tabular}

${ }^{a}$ Integrated over the $\mathrm{PV}{ }^{12} \mathrm{CO}(1-0)$ map shown in Fig. $4 ;{ }^{b}$ integrated over the $\mathrm{PdB}{ }^{12} \mathrm{CO}(1-0)$ map shown in Fig. $6 ;{ }^{c}$ not fully sampled.

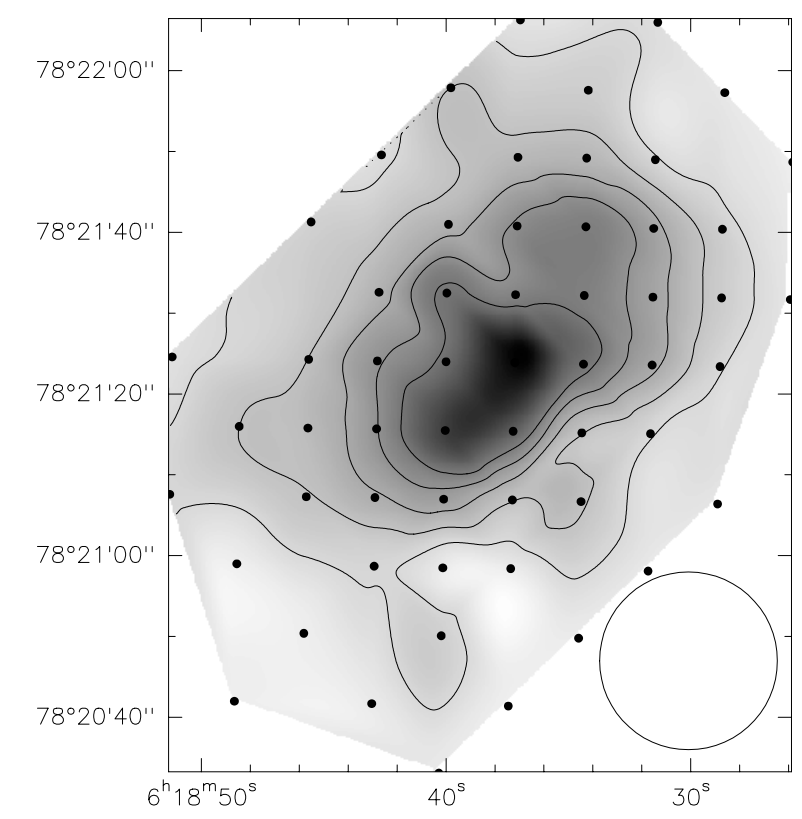

Fig. 4. ${ }^{12} \mathrm{CO}(1-0)$ observation with PV. The circle shows the beam width of 22" (FWHP); the dots the position of the observations. Contour levels at 20, 40, 60, 80, $100 \mathrm{~K} \mathrm{~km} \mathrm{~s}^{-1}$ /beam. Coordinates for J2000.

ultra-dense H II regions detected with MERLIN-VLA was published earlier (Greve et al. 2000). The map shows that these objects coincide mainly with the central $\mathrm{CO}$ concentration. The velocity-integrated emission of the ${ }^{12} \mathrm{CO}(1-0),{ }^{12} \mathrm{CO}(2-1)$, and ${ }^{13} \mathrm{CO}(1-0)$ line measured with $\mathrm{PdB}$ is shown in Fig. 6. From the integrated ${ }^{12} \mathrm{CO}(1-0)$ map of Fig. 6, we obtain the $\mathrm{CO}$ luminosity $L_{\mathrm{CO}(1-0)} \approx 0.6 \times 10^{9} \mathrm{~K} \mathrm{~km} \mathrm{~s}^{-1} \mathrm{pc}^{2}$. Compared with the CO luminosity derived from the PV single-dish observation (Table 3) we notice that the interferometer map misses $\sim 30 \%$ of the flux. This amount of CO may exist in a diffuse extended form.

The velocity-integrated maps (Fig. 6) of the optically-thick ${ }^{12} \mathrm{CO}$ line and the optically-thin ${ }^{13} \mathrm{CO}$ line (see Xie et al. 1994) reveal a warped distribution of $\sim 4 \mathrm{kpc}$ extent. This warp is not seen in either the $1.4 \mathrm{GHz}$ map by Condon et al. (1982) or the 8.3 GHz map by Zhao et al. (1996). Apparently, the warp is seen in the $15 \mathrm{GHz}$ synchrotron emission (Lisenfeld et al. 1996, their Fig. 1). The earlier proposed central S-shaped radio continuum emission of $\sim 2$ kpc extent (Kronberg \& Biermann 1981; Jackson \& Ho 1988; Zhao et al. 1996), which at the VLA-MERLIN angular resolution coincides with 3 individual sources (Kronberg \& Biermann 1981; Tarchi et al. 2000), is not seen in the molecular emission. The short-lived, though perhaps repetitive, firehose bending effect discussed in this context by Griv \& Chiueh (1998) is probably only seen in the disk of H I, if acting at all. 


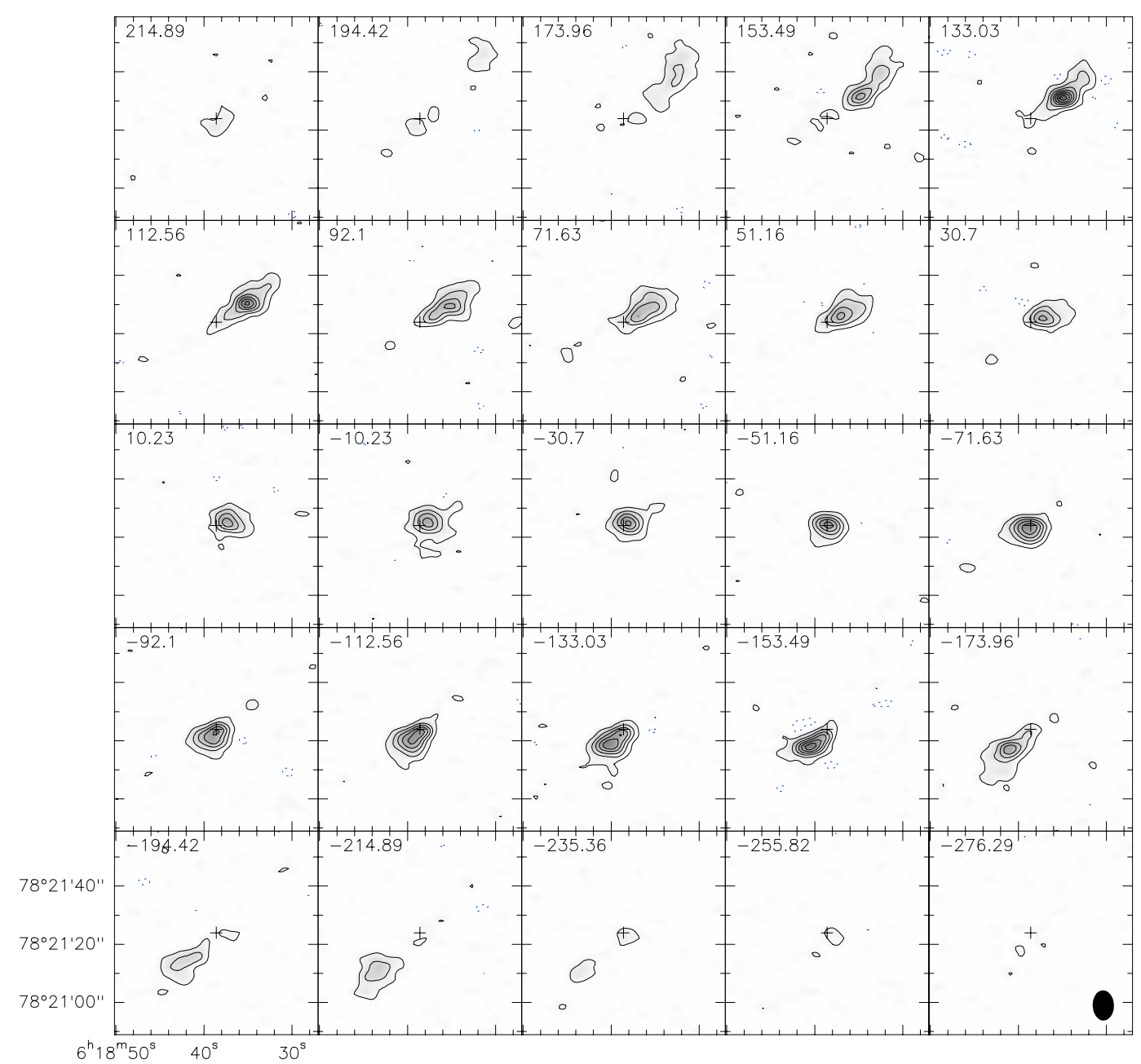

Fig. 5. Channel maps at the indicated velocities (minus the systemic velocity of $850 \mathrm{~km} \mathrm{~s}^{-1}$ ) of the ${ }^{13} \mathrm{CO}(1-0)$ emission measured with the $\mathrm{PdB}$ interferometer at $5.2^{\prime \prime} \times 3.6^{\prime \prime}[360 \mathrm{pc} \times 250 \mathrm{pc}$ ] resolution (beam in lower box). The contour levels are between 0.005 and $0.2 \mathrm{Jy} / \mathrm{beam}$, in steps of 0.005 . The cross is the center position given in footnote 1). Coordinates for J2000.

The feature of a warped gas distribution of a few $10^{9} M_{\odot}$ is, however, predicted in the $N$-body encounter calculations published by Mihos \& Hernquist (1996).

Figure 7 is the $\mathrm{CO}$ peak emission measured along the "projected warp plane". As evident from Fig. 6, the projected warp plane to the west bends away from the galactic plane towards the north, while to the east the projected warp plane bends to the south. On an extended, diffuse, and low-intensity $\mathrm{CO}$ background, three emission components are superimposed, i.e. a dominant central component (C) and the outer components $(1,2)$, which are interpreted as enhanced emission seen in the line-of-sight tangential direction of a weak molecular ring. The parameters of Gaussian profiles of these components are given in Table 4. The diameter of the molecular ring is $\sim 1.5 \mathrm{kpc}$, and its thickness in the galactic plane is $\sim 400 \mathrm{pc}(F W H M)$.

\section{Optical observations along the major axis}

The $4.2 \mathrm{~m}$ WHT was used in service time for a long-slit $\left(5^{\prime} \times\right.$ $\left.1.5^{\prime \prime}\right)$ observation along the major axis $\left(\mathrm{PA}=141^{\circ}\right)$ passing through the eye-estimated center of the galaxy. The true center is hidden behind dust with $A_{\mathrm{V}} \approx 4-7$ mag extinction (BCD-75; HEA-90; Smith et al. 1995) so that the exact position of the slit with respect to the center is uncertain. The observed lines are the emission lines $\mathrm{H} \alpha 6563 \AA$, [NII] 6548, $6583 \AA$, and [SII] 6716,
$6731 \AA$ in the visible, [SIII] $9069 \AA$ An the near-IR, and the stellar absorption lines of Ca II 8498, 8542, $8662 \AA$ in the near-IR. Radial velocities were determined from Gaussian profile fits to a precision of $\sim 30 \mathrm{~km} \mathrm{~s}^{-1}$, and the spatial sampling was similar to the seeing of $\sim 1^{\prime \prime}$. The derived radial velocities are used in Fig. 10 below. The spectroscopic observations do not allow the derivation of line widths, since a star was not observed to determine the instrumental profile.

\section{Kinematics}

The collection of previous and new data of the rotation is used to derive the mass of the starburst region.

\subsection{CO line velocities and line width}

Figure 8 is the velocity field of the molecular gas derived from the PdB ${ }^{12} \mathrm{CO}(1-0)$ observation. The figure displays the smooth and relatively undisturbed rotation of a galactic disk of $\sim \pm 300 \mathrm{pc}$ thickness (de-projected), seen close to edge-on. The center of rotation of the CO material coincides with the center of the dust and the stars. Channel maps of the $\mathrm{CO}$ emission (Greve et al. 2000) show low intensity streamers that were interpreted to indicate outflowing molecular material. 


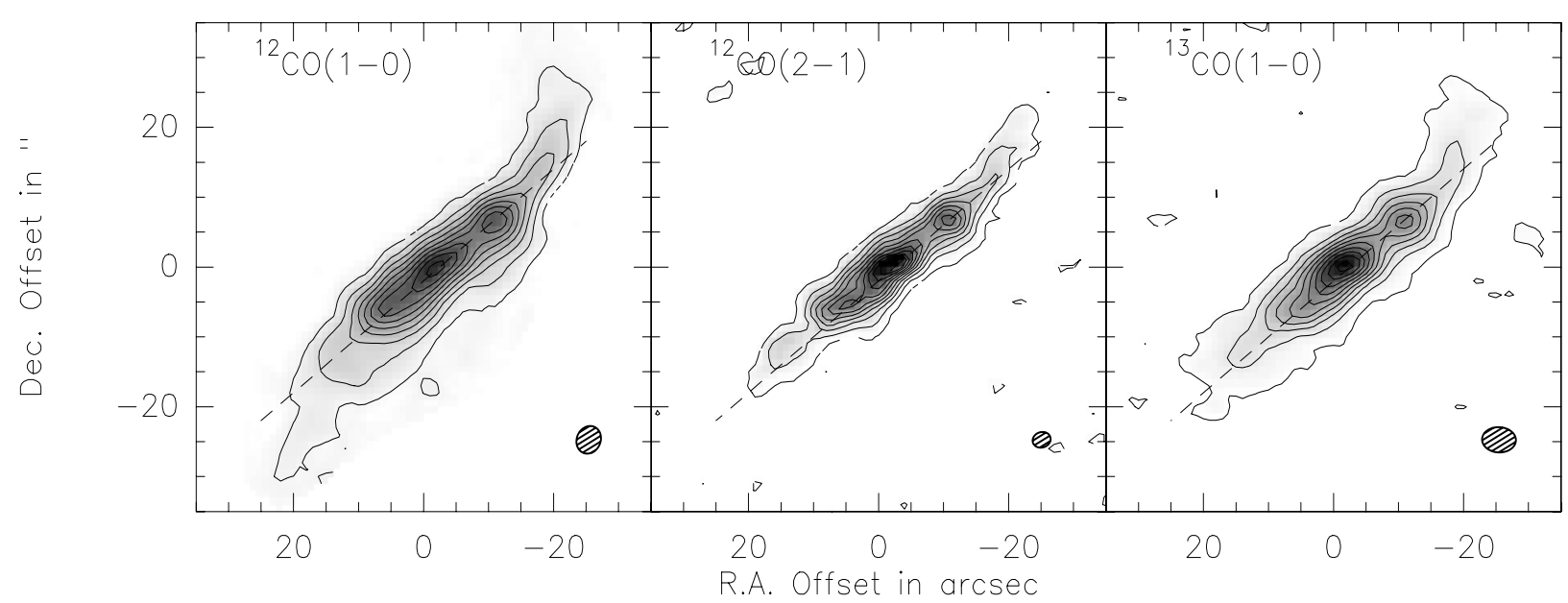

Fig. 6. PdB observation of velocity-integrated emission of ${ }^{12} \mathrm{CO}(1-0),{ }^{12} \mathrm{CO}(2-1)$, and ${ }^{13} \mathrm{CO}(1-0)$ (beams in the lower corner). Note that the warp is seen in the optically-thick ${ }^{12} \mathrm{CO}$ emission and the optically-thin ${ }^{13} \mathrm{CO}$ emission. The dashed line connects the emission peaks and extends approximately along the major axis $\left(\mathrm{PA}=145^{\circ}\right)$. The coordinate center is at RA $6^{\mathrm{h}} 18^{\mathrm{m}} 38.6^{\mathrm{s}}$, Dec $78^{\circ} 21^{\prime} 24.0^{\prime \prime}$ [2000]. Contour levels $($ above 2 sigma noise) for ${ }^{12} \mathrm{CO}(1-0): 5$ to $100 \mathrm{Jy} \mathrm{km} \mathrm{s}{ }^{-1}$ /beam by $10 ;{ }^{12} \mathrm{CO}(2-1): 1.75$ to $40 \mathrm{Jy} \mathrm{km} \mathrm{s} /$ beam by $4 ;{ }^{13} \mathrm{CO}(1-0): 0.35$ to $10 \mathrm{Jy} \mathrm{km} \mathrm{s}{ }^{-1} / \mathrm{beam}$ by 1 .

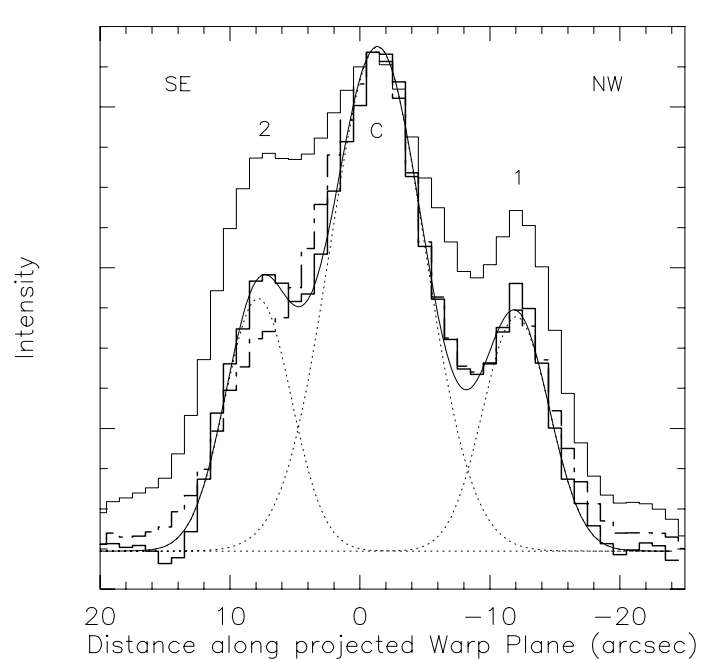

Fig. 7. CO distribution along the line-of-sight projected warp plane (normalized to the center). The histogram lines show the measured intensities (normalized to the peak intensity) of ${ }^{12} \mathrm{CO}(1-0)$ : thin line, ${ }^{12} \mathrm{CO}(2-1)$ : thick line, and ${ }^{13} \mathrm{CO}(1-0)$ : dashed line. The Gaussian fits are inserted as dotted lines and the continuous line.

Table 4. Gaussian components of the central $\mathrm{CO}$ emission (C) and of the line-of-sight tangential $\mathrm{CO}$ emission of the molecular ring $(1,2)$ (Fig. 7), measured with a beam of $\sim 300 \times 250$ pc width $(F W H M)$. For $d=14.5 \mathrm{Mpc}$.

\begin{tabular}{lccc}
\hline \hline Component & $\begin{array}{c}\text { Separation } \\
\text { from }(\mathrm{C})[\mathrm{pc}]\end{array}$ & $\begin{array}{c}\text { Extent }^{a} \\
(F W H M)[\mathrm{pc}]\end{array}$ & $I_{\mathrm{CO}} / I_{\mathrm{CO}}(\mathrm{C})^{b}$ \\
\hline NW (1) & 770 & $450(375)$ & $0.30 \pm 0.05$ \\
Center (C) & 0 & $650(600)$ & 1.0 \\
SE (2) & 650 & $500(430)$ & $0.4 \pm 0.1$ \\
\hline
\end{tabular}

${ }^{a}$ In brackets: deconvoled for the beam width; ${ }^{b}$ intensity ratio for peak of profile.

The high spatial resolution of the interferometer maps allows the derivation of the $\mathrm{CO}$ line width, to a large extent free of galactic rotation as it would affect low spatial-resolution, single-dish observations. Figure 9 is the ${ }^{12} \mathrm{CO}(1-0)$ line width $w$

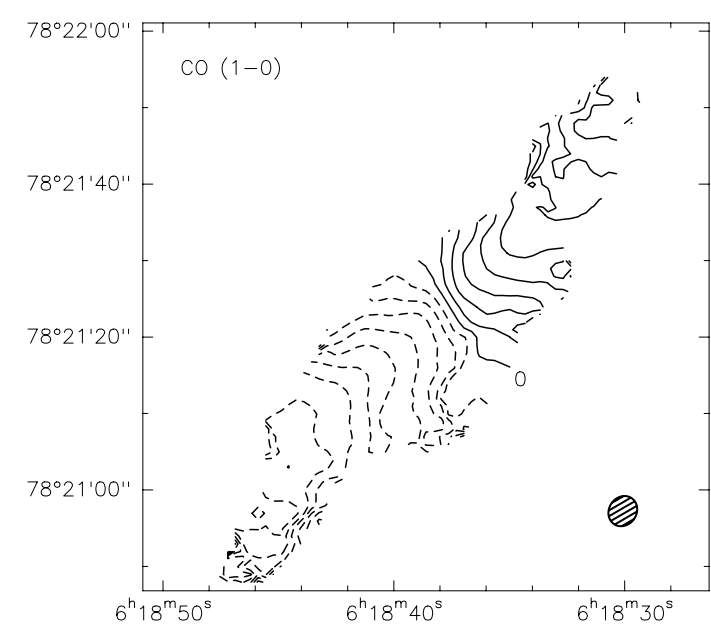

Fig. 8. PdB observation of ${ }^{12} \mathrm{CO}(1-0)$ radial velocities - systemic velocity $\left(850 \mathrm{~km} \mathrm{~s}^{-1}\right)$, contours in steps of $25 \mathrm{~km} \mathrm{~s}^{-1}$. The zero velocity is marked; solid lines: receeding velocities; dashed lines: approaching velocities. The displayed radial extent along the major axis is $r \approx \pm 3 \mathrm{kpc}$. Coordinates for $\mathbf{J} 2000$.

$[F W H M]$ measured along the projected warp plane. A broad component (A) associated with the starburst material exists, showing a gradual decrease in the line width with increasing distances from the center. The spatial extent of the approximated Gaussian component is $d(\mathrm{~A})=1.8 \mathrm{kpc}(F W H M)$, and the line width at the center is $w(0)=110 \mathrm{~km} \mathrm{~s}^{-1}\left(140 \mathrm{~km} \mathrm{~s}^{-1}\right.$ peak value $)$. From a Gaussian decomposition of the ${ }^{12} \mathrm{CO}(3-2)$ profile measured at the center of the galaxy, Dumke et al. (2001) find comparable line widths of $130 \mathrm{~km} \mathrm{~s}^{-1}$ and $150 \mathrm{~km} \mathrm{~s}^{-1}$, respectively. Approximately $1.3 \mathrm{kpc}$ to the NW, at the edge of the starburst region, a strong deviation from the regular distribution occurs (B). The line width here is $w(1.3 \mathrm{kpc})=140 \mathrm{~km} \mathrm{~s}^{-1}$, and the extent of the region is $d(\mathrm{~B})=450 \mathrm{pc}$.

\subsection{The central region radial velocities $(r \leq 4 \mathrm{kpc})$}

Figure 10a shows the radial velocities within $r \lesssim \pm 70^{\prime \prime}$ ( $\pm 5 \mathrm{kpc})$ distance from the center as derived from $\mathrm{H} \alpha$ line 


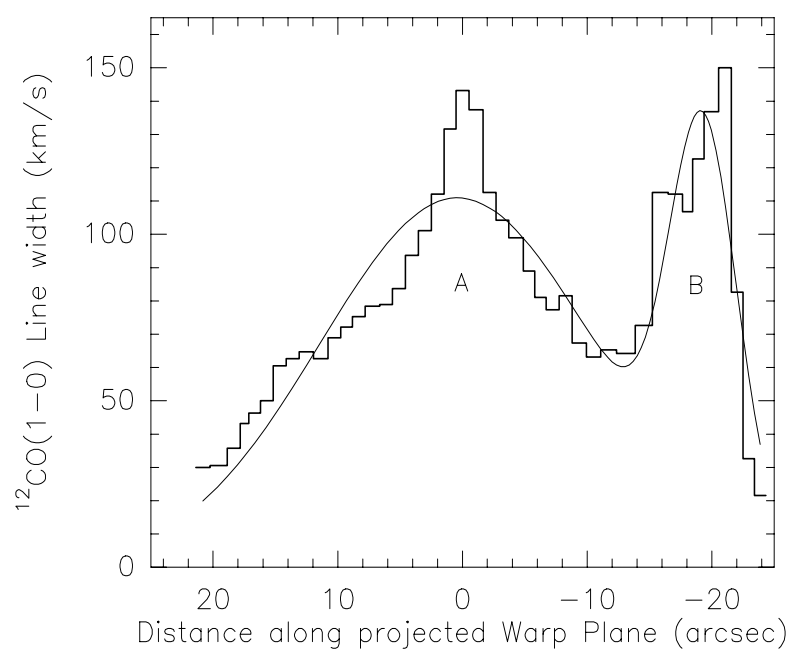

Fig. 9. PdB measured ${ }^{12} \mathrm{CO}(1-0)$ line width $(F W H M)$ : histogram line; Gaussian fit: solid line. Component $\mathrm{A}$ is associated with starburst material; B is perhaps a separate component.

observations by Burbidge et al. (1959; $\left.\mathrm{PA} \approx 141^{\circ}\right), \mathrm{BCD}-75$ $\left(\mathrm{PA} \approx 143^{\circ}\right)$, and from this observation $\left(\mathrm{PA}=141^{\circ}\right)$. The visible [NII] and [SII] lines, not shown here, reveal a similar velocity distribution. The figure suggests a solid body rotation of the starburst region $(r \lesssim \pm 2 \mathrm{kpc})$ and a velocity turn-over at $\sim \pm 250 \mathrm{~km} \mathrm{~s}^{-1}$ farther out. As already discussed by Prada et al. (1994), because of $A_{\mathrm{V}} \approx 4-7$ mag extinction towards the center and, in particular, towards the dust lanes, it is a priori not obvious whether the velocities derived from visible lines ( $\mathrm{H} \alpha$, [NII], [SII]) represent the rotation of the line-of-sight tangential directions. As illustrated in Fig. 10b, there is agreement between the radial velocities of the $\mathrm{H} \alpha$ line and the ${ }^{12} \mathrm{CO}$ and ${ }^{13} \mathrm{CO}$ lines, where the ${ }^{13} \mathrm{CO}$ line is optically thin.

Figure 10c shows the $\mathrm{H} \alpha$ velocities measured by HEA-90. At the SE side of the center a "dent" in the velocity curve seems to occur, which, however, is not clearly seen in the $\mathrm{H} \alpha$ observation of BCD-75. The velocities derived by Kobulnicky \& Gebhardt (2000) from the [OII] 3727, $3729 \AA$ line indicate a flatter rotation (Fig. 10c), as expected from gas seen farther away from the center because of higher extinction at near-UV wavelengths. Kobulnicky \& Gebhardt furthermore show that the [OII] emission lines and stellar Ca II H \& K aborption lines at $\mathrm{UV}$-wavelengths reveal the same rotation of gas and stars. This is also illustrated in Fig. 11, which shows the correlation of velocities derived from our near-infrared [SIII] emission line and stellar Ca II absorption line measurement. Over the central $\pm 600 \mathrm{pc}$ $\left(\sim \pm 10^{\prime \prime}\right)$ where the Ca II lines are seen, there is no difference in the rotation of H II region gas, thus of young stars and of the late-type stars seen in Ca II.

Figure 10d shows both the [SIII] line observation by Prada et al. (1994) and derived in this paper and the $\operatorname{Br} \gamma$ line observation of HEA-90. The [SIII] emission may still be affected by extinction, but the $\mathrm{Br} \gamma$ emission is not. The [SIII] velocities are steeper than the $\mathrm{H} \alpha$ velocities, while the $\mathrm{Br} \gamma$ observation shows a very steep rotation and a very large radial velocity difference of $\sim \pm 350 \mathrm{~km} \mathrm{~s}^{-1}$ over the distance of $\sim \pm 1 \mathrm{kpc}\left(\sim \pm 15^{\prime \prime}\right)$, as not seen in other lines.

Finally, the radial velocities derived from $\mathrm{H}$ I absorption-line measurements within the central $\pm 1.5 \mathrm{kpc}$ (Tarchi et al. 2004) agree with our and BCD-75's $\mathrm{H} \alpha$ line data and the $\mathrm{CO}$ line data, shown in Fig. 10.

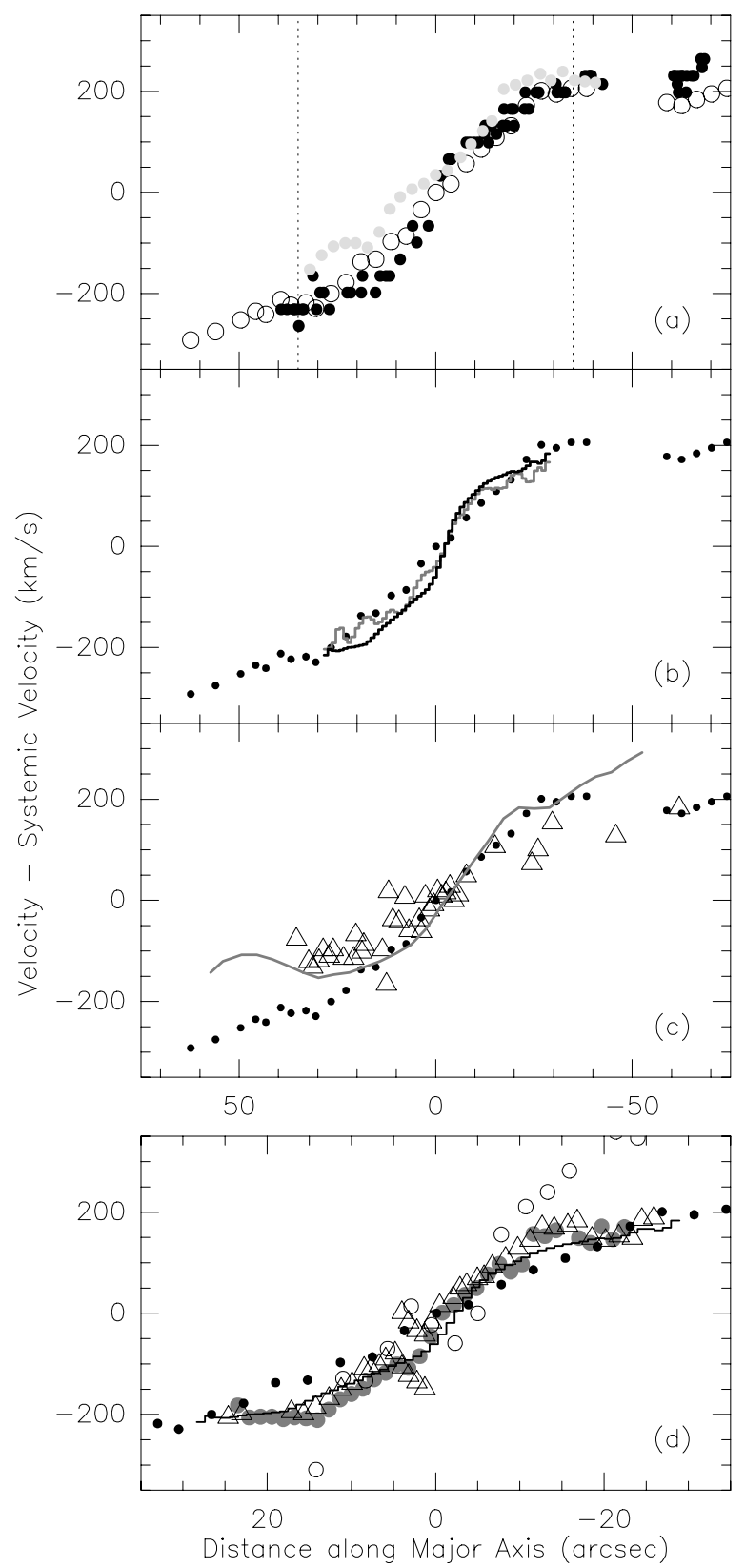

Fig. 10. Radial velocities - systemic velocity $\left(850 \mathrm{~km} \mathrm{~s}^{-1}\right)$. a) $\mathrm{H} \alpha$ emission line. This paper: solid dots; BCD-75: open circles; Burbidge et al. (1959): gray dots. The dashed lines show the extent of the stellar bar (Cabrera-Lavers \& Garzon 1994). b) ${ }^{12} \mathrm{CO}(1-0)$ (black line) and ${ }^{13} \mathrm{CO}(1-0)$ (gray line) emission lines this paper: histogram lines; for comparison the $\mathrm{H} \alpha$ emission from a) (BCD-75) is inserted: solid dots. c) $\mathrm{H} \alpha$ line from Hutchings et al. (1990) showing a velocity "dent" at SE: solid line; [OII] 3727, 3729 Å line from Kobulnicky \& Gebhardt (2000): triangles; $\mathrm{H} \alpha$ emission from a) (BCD-75): dots. d) [SIII] line from Prada et al. (1994): triangles, and this paper: gray dots; $\mathrm{Br} \gamma$ from Hutchings et al. (1990): open circles; $\mathrm{H} \alpha$ from a) (BCD-75): solid dots. The histogram line is for ${ }^{12} \mathrm{CO}(1-0)$. SE is to the left, NW to the right.

We have inserted in Fig. 10a the extent of the stellar bar, proposed from photometric measurements by Cabrera-Lavers \& Garzon (2004). The bar extends through the entire starburst region, but a kinematic signature is not evident. In the central region of the galaxy, an expanding bubble was noticed by Prada et al. (1994). However, this velocity feature is not seen in our data and hence may be of dubious nature. 


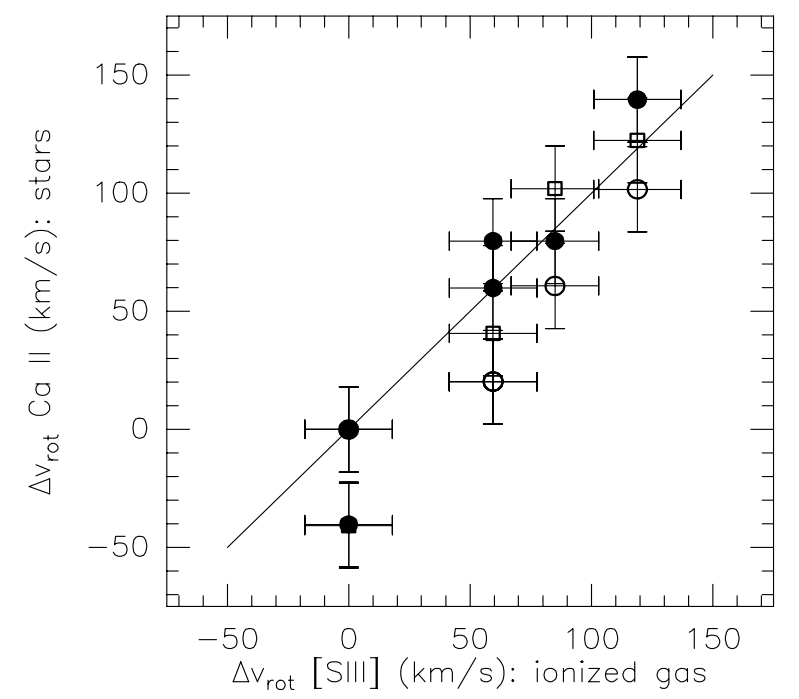

Fig. 11. Correlation (inside $\sim \pm 600 \mathrm{pc}$ distance from the center) of the radial velocity of ionized gas measured from the [SIII] $9069 \AA$ emission line and of late type stars measured from the Ca II absorption lines at $8662 \AA$ (solid dots), $8542 \AA$ (open circles), and $8496 \AA$ (squares). The straight line indicates a one-to-one correlation.

\subsection{Mass model $M(r)(r \leq 6 \mathrm{kpc})$}

For a density $\rho(r)=\rho_{\mathrm{o}} p(r)(0 \leq p \leq 1)$, as function of radial distance $r$, and a spheroidal mass distribution of eccentricity $e$, the rotation velocity $v(r)$, at $r$, and the mass $M(<r)$, within $r$, are given by (Burbidge et al. 1959, BCD-75)

$v^{2}(r)=4 \pi G \sqrt{1-e^{2}} \int_{o}^{r} \frac{\rho(\omega) \omega^{2} \mathrm{~d} \omega}{\sqrt{r^{2}-e^{2} \omega^{2}}}$

$M(<r)=4 \pi \sqrt{1-e^{2}} \int_{o}^{r} \rho(\omega) \omega^{2} \mathrm{~d} \omega$

with $G$ the gravity constant. There are three unknowns in these equations, viz. the eccentricity, the function $p(r)$, and the value $\rho_{\mathrm{o}}$ at the center of the galaxy. Burbidge et al. (1959) used $e=0.87(c / a=0.5)$, as suggested by optical images of the galaxy, and a bell-shaped density distibution $\rho(r)$ with the peak at $r_{\mathrm{o}} \approx 12^{\prime \prime}$ and $\rho\left(r_{\mathrm{o}}\right)=1.6 \times 10^{-22} \mathrm{~g} \mathrm{~cm}^{-3}=2 M_{\odot} \mathrm{pc}^{-3}$, and with a 100 times lower density at the center. On the other hand, BCD-75 use $e=0.995$, as suggested by the luminosity profile of a galactic disk, and $\rho(r)=\rho_{\mathrm{o}} \exp (\alpha r)$ and $\alpha=-0.05[r$ in arc sec], with $\rho_{\mathrm{o}}=5.85 \times 10^{-22} \mathrm{~g} \mathrm{~cm}^{-3} \approx 8 M_{\odot} \mathrm{pc}^{-3}$.

The velocity observations of $\mathrm{H} \alpha,[\mathrm{NII}]$, and [SII] interpreted by BCD-75 cover the radial extent of $\sim \pm 80^{\prime \prime}$ from the center. The data of the near-IR line [SIII] and the CO line are not investigated with the mass model, Eqs. (1) and (2). Figure 10d indicates that in the center region of $r \lessgtr 30-40^{\prime \prime}$ the [SIII] and $\mathrm{CO}$ lines show steeper rotation than the $\mathrm{H} \alpha$ line. Although there is some difference between the [SIII] and the $\mathrm{CO}$ rotation curves, when using the leading edges of the [SIII] rotation curve and keeping the exponential density distribution of BCD-75, we find that a slightly different value $\alpha=-0.0575$ gives a good fit to the steeper velocities. This is shown in Fig. 12. In essence, Eqs. (1) and (2) define the parameter $\sqrt{1-e^{2}} \rho_{\mathrm{o}}$ that has the value $\sim 1.2 \times$ $10^{-22} \mathrm{~g} \mathrm{~cm}^{-3}$ for $0.85 \lesssim e$ and $d=14.5 \mathrm{Mpc}$. The mass distribution $M(<r)$ is obtained from Eq. (2) using this value. The original value derived by BCD-75 (see Table 5) needs to be increased by $\sim 15 \%$, which is however within the errors of the analysis (distance, rotation curve).

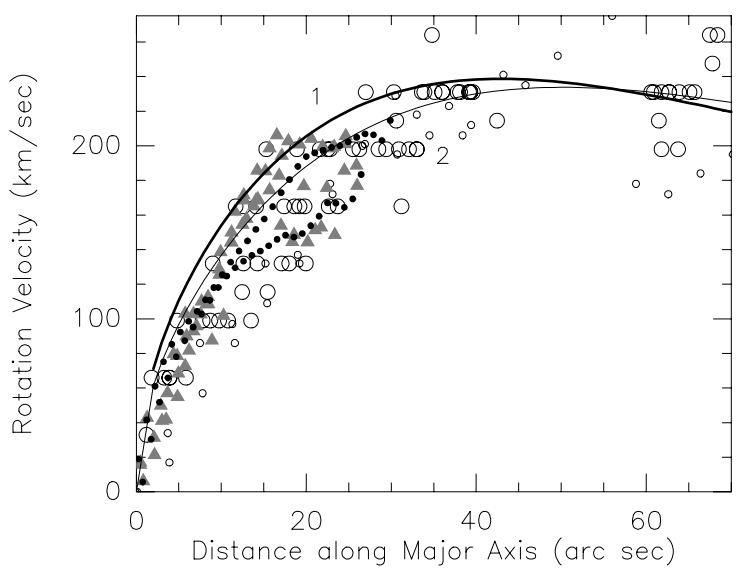

Fig. 12. Observed rotation velocity for a spheroidal mass distribution. Measured $\mathrm{H} \alpha$ velocities of $\mathrm{BCD}-75$ and this paper: open circles, [SIII] velocities from Prada et al. (1994) and this paper: triangles; ${ }^{12} \mathrm{CO}(1-0)$ line: dots; the velocities are folded around the center. The curve 1 is for $\alpha=-0.0575$, the curve 2 is for $\alpha=-0.05$ (BCD-75).

Table 5. Mass distribution of NGC 2146's starburst region, for $d=$ $14.5 \mathrm{Mpc}$.

\begin{tabular}{lcc}
\hline \hline Component & $\begin{array}{c}\text { Radius }(r) \\
{[\mathrm{kpc}]}\end{array}$ & $\begin{array}{c}\text { Mass }(<r) \\
{\left[M_{\odot}\right]}\end{array}$ \\
\hline${\text { Young stars }(\mathrm{OB})^{a}}^{a}$ & 2 & $\sim 10^{7}$ \\
Late-type stars $^{a}$ & 2 & $1 \times 10^{10}$ \\
Gas + dust $^{a}\left[\mathrm{M}\left(\mathrm{H}_{2}\right)\right]$ & 2 & $4.6 \times 10^{9}$ \\
Dynamic mass $^{a}$ & 2 & $1.6 \times 10^{10}$ \\
\hline Dynamic mass $^{b}$ & 2.8 & $1.7 \times 10^{10}$ \\
\hline
\end{tabular}

${ }^{a}$ This paper; ${ }^{b}$ Benvenuti et al. (1975).

The radial velocities shown in Figs. 10 and 12 are not corrected for the inclination $i$. The true radial velocity $v^{\prime}$ is related to the observed radial velocity $v$ by $v^{\prime}=v / \sin (i)$, with $i \approx 65^{\circ}$. The velocities $v^{\prime}$ are used to solve Eq. (1) and, subsequently, to calculate the mass from Eq. (2). For these values, the mass inside the starburst region then is $M(r<2 \mathrm{kpc})=(1.6 \pm 0.15) \times$ $10^{10} M_{\odot}$, for $d=14.5 \mathrm{Mpc}$.

\subsection{Mass of young stars}

The radial velocities give a dynamic mass of the starburst region of $M_{\mathrm{dyn}}(<2 \mathrm{kpc}) \approx 1.6 \times 10^{10} M_{\odot}$. When comparing the gas mass $M\left(\mathrm{H}_{2}\right)$ (Table 3$)$ and the dynamic mass $M_{\text {dyn }}(<2 \mathrm{kpc})$, we find that the mass of stars $M_{*}$ inside the starburst region is $M_{*} \approx M_{\mathrm{dyn}}-M\left(\mathrm{H}_{2}\right) \approx 1 \times 10^{10} M_{\odot}$. If we assume that the farIR luminosity of $L_{\mathrm{IR}}=6 \times 10^{10} L_{\odot}$ is mainly due to emission from dust heated by OB stars, of mass $m_{\mathrm{OB}} \approx 10-20 M_{\odot}$. and of luminosity $L_{\mathrm{OB}} \approx 10^{5} L_{\odot}$, then the present total mass of OB stars of the starburst region is on the order of $M_{\mathrm{OB}} \approx 10^{7} M_{\odot}$.

\section{5. $M\left(H_{2}\right) / M(H I)$ and $\left\langle n\left(H_{2}\right)\right\rangle /\langle n(H I)\rangle$}

Young et al. (1988) give $\log M\left(\mathrm{H}_{2}\right)=10.09\left(M_{\odot}\right)$ and $\log M(\mathrm{HI})=10.10\left(M_{\odot}\right)$, thus $M\left(\mathrm{H}_{2}\right) / M(\mathrm{HI}) \approx 1$, with $M\left(\mathrm{H}_{2}\right)$ based on $\mathrm{CO}$ observations and $M(\mathrm{HI})$ derived from data collected by Huchtmeier \& Richter (1989). Taramopoulos et al. (2001) derive a $\mathrm{H}$ I mass for the region of the galaxy of $M(\mathrm{H} \mathrm{I})=$ $1.6 \times 10^{9} M_{\odot}$. The same value is found by Fisher \& Tully (1976) when using $d=14.5 \mathrm{Mpc}$, and their assumption that $\sim 60 \%$ of the 
Table 6. Geometrical alignment of stellar and gaseous components.

\begin{tabular}{|c|c|c|c|c|c|c|}
\hline Component & $\begin{array}{l}\text { Major axis }{ }^{a} \\
\text { (2a) }[\mathrm{kpc}]\end{array}$ & $\begin{array}{c}\text { Ratio }^{b} \\
\text { b/a }\end{array}$ & $\begin{array}{c}\text { PA } \\
\text { axis a }\left[^{\circ}\right]\end{array}$ & $\begin{array}{c}\mathrm{RA}(2000)^{c} \\
{[\mathrm{~h} \mathrm{~m} \mathrm{~s}]}\end{array}$ & $\begin{array}{c}\operatorname{Dec}(2000)^{c} \\
{\left[{ }^{0},{ }^{\prime} 1\right.}\end{array}$ & Ref. \\
\hline \multicolumn{7}{|l|}{ Starburst region } \\
\hline$R$-image & 6 & 0.50 & 135 & 61836.4 & 782124.0 & (1) (their Fig. 4) \\
\hline$K$-image & 3.5 & 0.40 & 130 & 61837.9 & 782124 & (2) \\
\hline Stellar bar & 5 & 0.56 & 138 & & & (3) \\
\hline CO emission (peak) & 3 & 0.30 & 130 & 61838.0 & 782124 . & Fig. 6, warp \\
\hline Warp direction $(\mathrm{CO})$ & & & $\sim 115$ & & & Fig. 6 \\
\hline Dust emission $(230 \mathrm{GHz})$ & 4 & 0.45 & 130 & 61837.5 & 782123 . & Fig. 1 \\
\hline Dustlanes & & & $150-190$ & & & (1) \\
\hline Optical polarization & & 0.6 & 135 & & & (4) (their Fig. 1) \\
\hline $15 \mathrm{GHz}$ synchrotron emission & 4.5 & 0.35 & 130 & 61839.5 & 782122.5 & (5) (their Fig. 1; warp) \\
\hline \multicolumn{7}{|l|}{ Large-scale features (stars) } \\
\hline Feature I & 22 & 0.3 & 120 & 61846 & 782140 & Fig. 13 \\
\hline Feature II & 14 & 0.3 & 150 & 61834 & 782140 & Fig. 13 \\
\hline \multicolumn{7}{|l|}{ Large-scale features (gas) } \\
\hline H II region ring ${ }^{d}$ (Feature III) & 20 & 0.55 & 145 & 6183 & 782046 & (1) (their Fig. $4 ; \mathrm{H} \alpha$ ) \\
\hline H I Southern tail ${ }^{d}$ & 85 & 0.45 & 160 & $\sim 614$ & $\sim 781$ & $(6),(7)$ \\
\hline \multicolumn{7}{|l|}{ Other components } \\
\hline Central radio source & & & & 61837.6 & 782124.2 & (4) \\
\hline Center of rotation & & & & 61837.5 & 782123 & Fig. 8 \\
\hline
\end{tabular}

${ }^{a}$ Dimension (2a) of longest extension; ${ }^{b}$ extension (2b) in perpendicular direction, not de-projected for inclination; ${ }^{c}$ center position; ${ }^{d}$ incomplete feature.

References: (1) Young et al. (1988); (2) 2MASS: www .irsa.ipac. caltech.edu; (3) Cabrera-Lavers \& Garzon (2004); (4) Draper et al. (1995); (5) Lisenfeld et al. (1996); (6) Casper \& Shane (1996); (7) Taramopoulos et al. (2001).

measured $\mathrm{H}$ I mass is concentrated in the center region. For a gas mass of $M\left(\mathrm{H}_{2}\right)=4.6 \times 10^{9} M_{\odot}$, as derived above, this gives as crude estimate $M\left(\mathrm{H}_{2}\right) / M(\mathrm{HI}) \approx 3$.

Following Figs. 1 and 4, the volume of the dust and $\mathrm{H}_{2}$ emission of $\sim 4 \mathrm{kpc}$ diameter and of $\sim 1 \mathrm{kpc}$ de-projected total thickness ( $z$-direction) is $V \approx(0.5-1.2) \times 10^{10} \mathrm{pc}^{3}$, with the lower limit taking into account the possibility of an oval distribution of these axial dimensions. The average gas density in this region then is $\rho \approx M\left(\mathrm{H}_{2}\right) / V \approx 5 \times 10^{9} M_{\odot} /(0.5-1.2) \times 10^{10} \mathrm{pc}^{3} \approx$ $(0.5-1) M_{\odot} / \mathrm{pc}^{3} \approx 3 \times 10^{-23} \mathrm{gr} \mathrm{cm}^{-3}$ or, since primarily consisting of $\mathrm{H}_{2},\left\langle n\left(\mathrm{H}_{2}\right)\right\rangle \approx 10 \mathrm{~cm}^{-3}$. MERLIN-VLA H I line absorption measurements towards the central continuum source (Tarchi et al. 2000, 2004) indicate an H I column density of $\sim(1-3) \times$ $10^{22} \mathrm{~cm}^{-2}$. If we assume a line-of-sight distance towards the continuum source of 3-5 kpc and a homogeneous $\mathrm{HI}$ distribution, then the average $\mathrm{HI}$ density is $\langle n(\mathrm{HI})\rangle \approx 1 \mathrm{~cm}^{-3}$, so that $\Gamma=$ $\left\langle n\left(\mathrm{H}_{2}\right\rangle /\langle n(\mathrm{HI})\rangle \approx 10\right.$. The low average density $\left\langle n\left(\mathrm{H}_{2}\right)\right\rangle$ does not contradict the high density $n\left(\mathrm{H}_{2}\right) \approx 10^{4} \mathrm{~cm}^{-3}\left(\right.$ at $\left.T_{\mathrm{d}} \approx 55 \mathrm{~K}\right)$ derived from CO measurements (Xie et al. 1994; Mauersberger et al. 1999). If the average density $\left\langle n\left(\mathrm{H}_{2}\right)\right\rangle$ is entirely due to dense $n\left(\mathrm{H}_{2}\right)$ associated with molecular clouds, then a volume filling factor of $f_{\mathrm{v}} \approx\left\langle n\left(\mathrm{H}_{2}\right)\right\rangle / n\left(\mathrm{H}_{2}\right) \approx 0.001$ to 0.0005 would result. Such a value seems compatible with the beam-filling factor of $f_{\mathrm{B}} \approx 0.005$ derived by Xie et al. Table 5 summarizes the masses of the starburst region.

\section{Large-scale structures and velocities}

The spatial distribution of the starburst region material and their center positions are summarized in Table 6 . We observe that the material is well-ordered and that the gas and dust are aligned to the stars seen in the $K$-image (2MASS) and $R$-image (partially obscured by the dust lanes) published by YEA-88. The morphology of the starburst region does not provide an outstanding trace of an encounter, or merger, so that the evidence remaining from such an event may also be searched for in the outer regions where distortions of the galaxy are seen. The inspection of published material reveals three large-scale features, shown in Fig. 13 and included in Table 6. Two of these features (I, II) give the impression of stellar (sub)systems; the other feature (III) is an incomplete ellipse of $\mathrm{H}$ II regions and probably also of stars.

\subsection{Stellar features I and II}

On deep images (for instance YEA-88, Sandage \& Bedke 1994), the starburst region is surrounded by two arc-like features, partially forming the spiral arms described by BCD-75. These features are visible in continuum emission and do not contain prominent H II regions and young objects, unless they are heavily obscured. These features are 2 to 3 times larger than the starburst region. We assume that this material is approximately outlined, or confined, in the ellipsoidal distributions derived from the faint outer contours seen in the POSS image and shown in Fig. 13. The features I, II resemble stellar sub-systems, which are not co-planar. In the past, these systems may have been independent. With respect to the features I and II, the starburst occurs, approximately, inside the inner ellipse with center at $C_{*}$ as derived from the positions given in Table 6 . As is evident from the table, this center is also the center of the stars seen on the $R$ and $K$-band images.

\subsection{The incomplete ring of $\mathrm{H}$ II regions (feature III)}

The images published by Young et al. (1988) reveal an incomplete, though prominent ellipse of $\mathrm{H}$ II regions, associated in the NW with diffuse emission originating from stars (see Devereux et al. 1994). This incomplete ellipse of H II regions and its center at $\mathrm{C}_{\mathrm{III}}$ is reproduced in Fig. 13. We note that the center of the stellar body and the starburst region $\left(\mathrm{C}_{*}\right)$ and of the ellipse of $\mathrm{H}$ II regions $\left(\mathrm{C}_{\mathrm{III}}\right)$ is offset by the projected distance $\mathrm{C}_{\mathrm{III}}-\mathrm{C}_{*} \approx 5.5 \mathrm{kpc}$.

The apparent axial ratio of the ellipse and its orientation agrees with the other components of the galaxy, in particular 


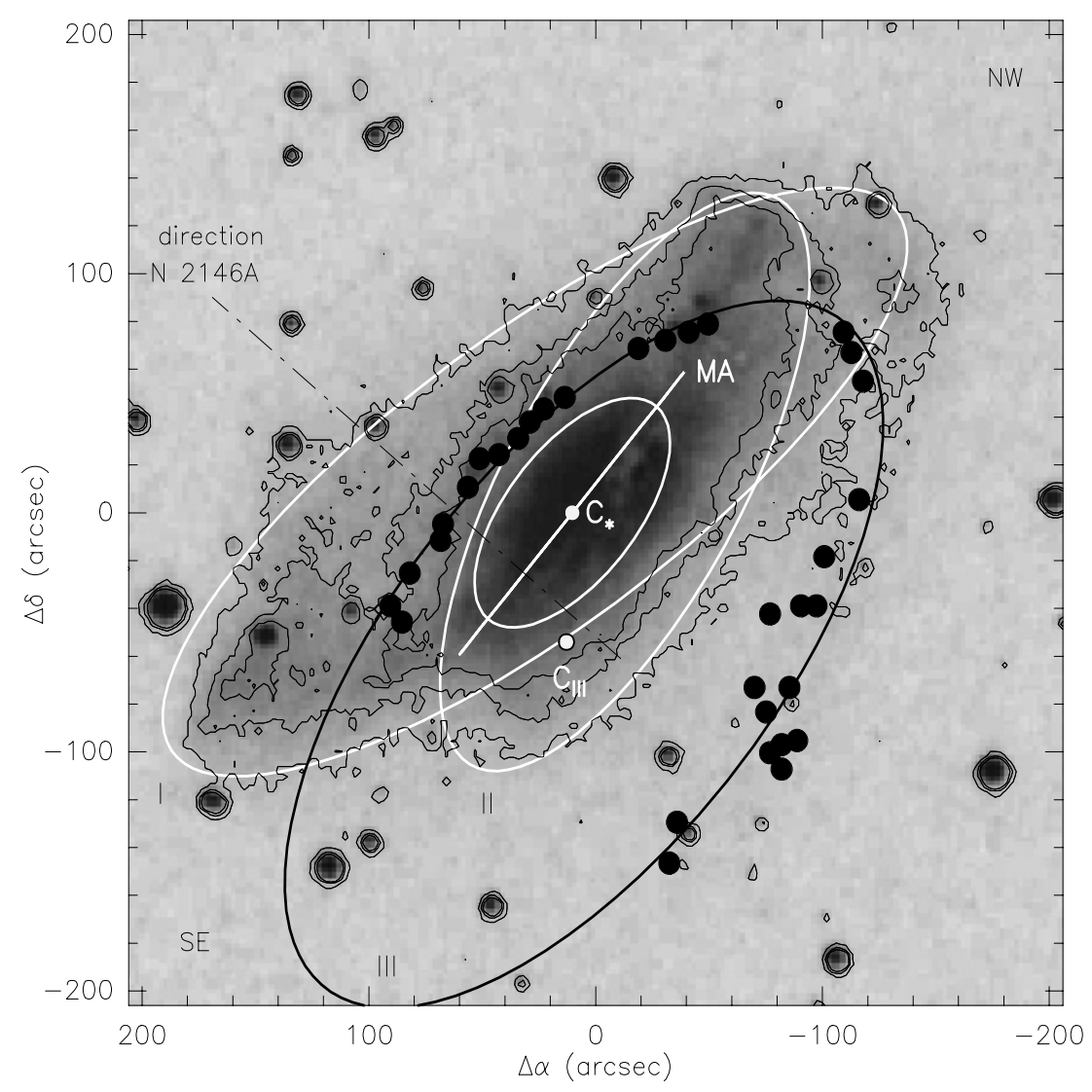

Fig. 13. Proposed morphology of NGC 2146 (digitized POSS). The inner ellipse with center at $C_{*}$ is the region of the starburst, with major axis MA. The ellipses I and II confine stellar concentrations, as also outlined by the low-level brightness contours (black lines). The ellipse III is the incomplete ring of $\mathrm{H}$ II regions (black dots; taken from Young et al. 1988) with center at $\mathrm{C}_{\mathrm{III}}$. For the coordinates of the centers $\mathrm{C}_{*}$ and $\mathrm{C}_{\mathrm{III}}$ see Table 6. $100^{\prime \prime}$ equals $\sim 7 \mathrm{kpc}$.

those of the starburst region (Table 6). On this basis we assume that the incomplete ellipse of $\mathrm{H}$ II regions is in fact an incomplete ring, seen inclined in a similar way as NGC 2146 by the angle $i=60-65^{\circ}$. The radius of the ring is $R_{\mathrm{III}} \approx 10 \mathrm{kpc}$. Under this assumption we apply the velocity analysis of an inclined ring, published by Few et al. (1982). Using the radial velocities $v$ of the $\mathrm{H}$ II regions measured by YEA-88 in their relation (5), we are able to derive the systemic velocity $v_{\text {sys }}$ of the ring, its rotational velocity $v_{\text {rot }}$, and its expansion velocity $v_{\text {exp }}$ from

$v=v_{\mathrm{sys}}-\left(v_{\mathrm{rot}} \cos \alpha+v_{\mathrm{exp}} \sin \alpha\right) \sin i$

with $\alpha=\tan ^{-1}[\tan \theta / \cos i]$, where $\theta$ is the angle between the ellipse semi-major axis and the direction of the selected H II region. The best-fit solution for the observed velocities of $9 \mathrm{H}$ II regions gives $v_{\text {sys }} \approx 880 \mathrm{~km} \mathrm{~s}^{-1}, v_{\text {rot }} \approx 270 \mathrm{~km} \mathrm{~s}^{-1}$, and $v_{\exp } \approx$ $35 \mathrm{~km} \mathrm{~s}^{-1}$. If we do not allow for expansion, the rotation velocity is $v_{\text {rot }}=220 \mathrm{~km} \mathrm{~s}^{-1}$, with $v_{\text {sys }} \approx 905 \mathrm{~km} \mathrm{~s}^{-1}$. When using a different angle $i^{\prime}$ with $15^{\circ} \lesssim\left|i-i^{\prime}\right|$ in Eq. (3), the calculated rotation velocity will be higher by $100 \mathrm{~km} \mathrm{~s}^{-1}$ or more. A ring with expansion gives a slightly better fit to the observation. If the rotation occurs around a centralized mass, an estimate of the mass is obtained from $v_{\text {rot }} \approx \mathrm{G} \mathrm{M} / r_{\text {III }}$, which gives $M \approx(1-3) \times 10^{11} M_{\odot}$.

We assume that the ring of $\mathrm{H}$ II regions is associated with the NGC 2146 complex, and we expect that the systemic velocity of the ring is similar to the systemic velocity of the galaxy, as the analysis of the velocity components (9) confirms. However, the ring apparently rotates around the center $\mathrm{C}_{\mathrm{III}}$, which is offset by $\sim 5.5 \mathrm{kpc}$ from the dynamic center $\mathrm{C}_{*}$ of the starburst region. The rotation of the ring behaves as if it were independent of the mass concentration in the starburst region.

Finally, we assume that the possible expansion of the ring of $\mathrm{H}$ II regions is related to some event, for instance, the interaction of NGC 2146 with another galaxy, which occurred some time $t_{\text {in }}$ ago. In the case where the present-day expansion velocity of $v_{\exp } \approx 35 \mathrm{~km} \mathrm{~s}^{-1}$ acted for most of the time, and from the dimension of the H II ring of $R_{\mathrm{III}} \approx 10 \mathrm{kpc}$, we arrive at a time $t_{\text {in }} \approx R_{\text {III }} / v_{\text {exp }} \approx 10 \mathrm{kpc} / 35 \mathrm{~km} \mathrm{~s}^{-1} \approx 300 \mathrm{Myr}$. Such an age is closer to a starburst occurring $100 \mathrm{Mr}$ after a merger, as predicted by Mihos \& Hernquist (1996) and Barnes \& Hernquist (1996), than the $1000 \mathrm{Myr}$ of an encounter postulated by Taramopoulos et al. (2001). However, the apparent coincidence or discrepancy rests entirely on the assumption of the inclination of the ring and the derived expansion.

\section{Summary}

The additional observations presented here confirm the regular geometry and kinematics of the starburst region in NGC 2146, with no outstanding evidence of an encounter or merger. The radio continuum measurements and the $\mathrm{CO}$ measurements indicate a gas mass of $M\left(\mathrm{H}_{2}\right)=4.6 \times 10^{9} M_{\odot}$ in the starburst region of $\sim 4 \mathrm{kpc}$ extent, while the dynamic mass of the starburst region is $\sim 1.6 \times 10^{10} M_{\odot}$. The molecular gas (CO) has a warped structure. The line width of the molecular gas $(\mathrm{CO})$ is abnormally wide over a $\sim 500 \mathrm{pc}$ wide region in the NW part of the starburst region. 
The outer region of the galaxy at $\sim 10 \mathrm{kpc}$ radial distance, or more, seems to consist of two stellar sub-systems, which are not co-planar, giving the impression of having been independent. These sub-systems may be interacting; the starburst occurs near the common center of the systems. A third system is an incomplete and, possibly, expanding ring of $\mathrm{H}$ II regions, and stars. The ring rotates around a center that is offset from the starburst region by $\sim 5.5 \mathrm{kpc}$. From the expansion of the ring we derive a possible age of $300 \mathrm{Myr}$.

This publication does not give an answer to the question of an encounter or a merger. However, the analysis of the motion of the ring of $\mathrm{H}$ II regions may give an indication that a decisive event has taken place some $\sim 300$ Myr ago. The ongoing starburst may be related to this event. Although this age seems closer to a starburst following $100 \mathrm{Myr}$ after a merger, as predicted by Mihos \& Hernquist (1996) and Barnes \& Hernquist (1996), rather than the age of an encounter 1000 Myr ago, as postulated by Taramopoulos et al. (2001), the assumptions involved in the interpretation of the observations do not allow one to decide between both hypotheses.

Acknowledgements. We are thankful for the optical observations in service time with the William Herschel Telescope (WHT) operated on the Island of La Palma by the INT Group in the Spanish Observatorio de Roque de los Muchachos of the Instituto de Astrofisica de Canarias.

We thank the referee for extensive and very critical comments, which also eliminated unjustified speculation; we appreciated his/her patience of dealing with this paper, and with us. The referee also suggested a closer investigation of the mass model.

\section{Appendix}

The flux density measured with the bolometer at $230 \mathrm{GHz}$ (Sect. 2.1) contains a contribution from synchrotron radiation, ${ }^{12} \mathrm{CO}(2-1)$ emission, and free-free emission. We estimate their respective contributions.

1. Synchrotron radiation. Following McCutcheon (1973), the integrated synchrotron emission of NGC 2146 is $S(6.6)=$ $0.35 \pm 0.03 \mathrm{Jy}$ at $6.6 \mathrm{GHz}$. With a spectral index of $\alpha=-0.74$, this gives a flux density at $230 \mathrm{GHz}$ of $S(230)=(6.6 / 230)^{0.74} \times 0.35=0.025 \mathrm{Jy}$.

2. ${ }^{12} \mathrm{CO}(2-1)$ emission. We follow Thuma et al. (2001) to estimate the ${ }^{12} \mathrm{CO}(2-1)$ contribution in the bolometer observation. At the center of the continuum emission, we have $S^{\text {bol }}=S_{\mathrm{c}} \times \Delta v \approx 0.087(\mathrm{Jy} /$ beam $) \times 60(\mathrm{GHz}) \approx$ $5 \times 10^{-17} \mathrm{~W} \mathrm{~m} \mathrm{~m}^{-2} /$ beam (the bandwidth may not be as large as $80 \mathrm{GHz}$, as used by Thuma et al., but around $60 \mathrm{GHz})$. For the central $\mathrm{CO}(2-1)$ emission we find $S^{\mathrm{CO}}=$ $150 \mathrm{~K} \mathrm{~km} \mathrm{~s}^{-1} /$ beam $=120 \mathrm{~K} \mathrm{MHz} /$ beam. Using $T_{\mathrm{mb}} \approx T_{\mathrm{ex}}$ in the same way, the Rayleigh-Jeans approximation gives $S^{\mathrm{CO}}=$ $8 \times 10^{-18} \mathrm{~W} \mathrm{~m} \mathrm{~m}^{-2} /$ beam, so that $S^{\mathrm{CO}} / S^{\text {bol }}=8 \times 10^{-18} / 5 \times$ $10^{-17} \approx 0.1-0.15$. We assume that this ratio holds throughout the entire starburst region so that the total contribution from $\mathrm{CO}$ is $\sim 0.04 \mathrm{Jy}$.

3. Free-free emission. There is no direct measurement of the free-free emission. With recourse to Zhao et al. (1996), RRL observation and model calculation (model A, Table 6), for a region of $\sim 10^{\prime \prime}$ the ratio of the thermal and non-thermal $(\alpha=-0.76)$ emission at $5 \mathrm{GHz}$ is $S_{\mathrm{th}} / S_{\mathrm{nth}}=5.9 \mathrm{mJy} / 69 \mathrm{mJy}=$ 0.08 . Assuming that this ratio holds throughout the starburst region, we have $S_{\text {nth }}=0.35 \mathrm{Jy}$ at $6.6 \mathrm{GHz}$ so that $S_{\text {th }} \approx$ $0.08 \times 0.35=0.03 \mathrm{Jy}$. Although the free-free emission has a slightly decreasing spectrum towards shorter wavelengths, we use $S_{\text {th }}=0.03 \mathrm{Jy}$.
The total contribution can be on the order of $0.025+0.04+$ $0.03 \sim 0.1 \mathrm{Jy}$, so that the corrected bolometer flux density is $S_{1.3}=0.36 \pm 0.025$.

\section{References}

Aalto, S., Booth, R. S., Black, J. H., \& Johansson, L. E. B. 1995, A\&A, 300, 369 Armus, L., Heckman, T. M., Weaver, K. A., \& Lehnert, M. D. 1995, ApJ, 445, 666

Barnes, J. E., \& Hernquist, L. 1996, ApJ, 471, 115

Benvenuti, P., Capaccioli, M., \& D’Odorico, S. 1975, A\&A, 41, 91 (BCD-75) Burbidge, E. M., Burbidge, G. R., \& Prendergast, K. H. 1959, ApJ, 130, 739

Cabrera-Lavers, A., \& Garzon, F. 2004, AJ, 127, 1386

Caspers, H. C. M., \& Shane, W. W. 1986, in Light on Dark Matter, 1st IRAS Conf., 445

Condon, J. J., Condon, M. A., Gisler, G., \& Puschell, J. J. 1982, ApJ, 252, 102 de Vaucouleurs, G. 1950, Ann. Astrophys., 13, 362

Della Ceca, R., Griffiths, R. E., Heckman, T. M., Lehnert, M. D., \& Weaver, K. A. 1999, ApJ, 514, 772

Devereux, N., Taniguchi, Y., Sanders, D. B., Nakai, N., \& Young, J. S. 1994, AJ, 107,2006

Draper, P. W., Done, C., Scarrott, S. M., \& Stochdale, D. P. 1995, MNRAS, 277, 1430

Dumke, M., Nieten, Ch., Thuma, G., Wielebinski, R., \& Walsh, W. 2001, A\&A, 373,853

Few, J. M. A., Madore, B. F., \& Arp, H. C. 1982, MNRAS, 199, 633

Fisher, J. R., \& Tully, R. B. 1976, A\&A, 53, 397

Greve, A., Neininger, N., Tarchi, A., \& Sievers, A. 2000, A\&A, 364, 409

Griffin, M. J., \& Orton, G. S. 1993, Icarus, 105, 537

Griv, E., \& Chiueh, T. 1998, ApJ, 503, 186

Huchtmeier, W. K., \& Richter, O.-G. 1989, A General Catalog of H I Observations of Galaxies (New York, Heidelberg: Springer Verlag)

Hutchings, J. B., Lo, E., Neff, S. G., Stanford, S. A., \& Unger, S. W. 1990, AL, 100, 60 (HEA-90)

Inui, T., Matsumoto, H., Tsuru, T. G., et al. 2005, PASJ, 57, 135

Jackson, J. M., \& Ho, P. T. P. 1988, ApJ, 324, L 5

Kobulnicky, H. A., \& Gebhardt, K. 2000, AJ, 119, 1608

Kronberg, P. P., \& Biermann, P. 1981, ApJ, 243, 89

Lisenfeld, U., Alexander, P., Pooley, G. G., \& Wilding, T. 1996, MNRAS, 281, 301

Mauersberger, R., Guelin, M., Martin-Pintado, J., et al. 1989, A\&AS, 79, 217

Mauersberger, R., Henkel, C., Walsh, W., \& Schulz, A. 1999, A\&A, 341, 256

McCutcheon, W. H. 1973, AJ, 78, 18

Mezger, P. G., Wink, J. E., \& Zylka, R. 1990, A\&A, 228, 95

Mihos, J. C., \& Hernquist, L. 1996, ApJ, 464, 641

Prada, F., Beckman, J. E., McKeith, C. D., Castles, J., \& Greve, A. 1994, ApJ, 423, L 35

Rickard, L. J., \& Harvey, P. M. 1984, AJ, 89, 1520

Roberts, M. S. 1978, AJ, 83, 1026

Sandage, A., \& Tammann, G. A. 1987, in A Revised Shapley-Ames Catalog of Bright Galaxies (Carnegy Institution of Washington Publication), Publ. No. 637, 2nd edn.

Sandage, A., \& Bedke, J. 1994, The Carnegie Atlas of Galaxies, Carnegie Institution with the Flintridge Foundation, 1, 146, Washington DC, USA

Sanders, D. B., Soifer, B. T., Neugebauer, G., et al. 1986, ApJ, 289, 129

Sanders, D. B., Mazzarella, J. M., Kim, D.-C., Surace, J. A., \& Soifer, B. T. 2003, AJ, 126, 1607

Sanders, D. B., Scoville, N. Z., \& Soifer 1991, ApJ, 370, 158

Schmitt, H. R. 2001, AJ, 122, 2243

Smith, B. J., Harvey, P. M., \& Lester, D. F. 1995, ApJ, 442, 610

Surace, J. A., Sanders, D. B., \& Mazzarella, J. M. 2004, AJ, 127, 3235

Taramopoulos, A., Payne, H., \& Briggs, F. H. 2001, A\&A, 365, 360

Tarchi, A., Neininger, N., Greve, A., et al. 2000, A\&A, 358, 95

Tarchi, A., Henkel, C., Peck, A. B., \& Menten, K. M. 2002, A\&A, 389, 39

Tarchi, A., Greve, A., Peck, A. B., et al. 2004, MNRAS, 351, 339

Thuma, G., Neininger, N., Klein, U., \& Wielebinski, R. 2000, A\&A, 358, 65

Wallin, J. F., \& Stuart, B. V. 1992, ApJ, 399, 29

Xie, S., Young, J. S., \& Schloerb, F. P. 1994, ApJ, 421, 434

Young, J. S., Claussen, M. J., Kleinmann, S. G., Rubin, V., \& Scoville, N. 1988, ApJ, 331, L 81 (YEA-88)

Young, J. S., Xie, S., Kenney, J. D. P., \& Rice, W. L. 1989, ApJS, 70, 699

Zhao, J.-H., Anantharamaiah, K. R., Goss, W. M., \& Viallefond, F. 1996, ApJ, 472,54 